\title{
Hiệu ứng GARCH trên dãy lợi suất thị trường chứng khoán Việt Nam 2000-2003
}

\author{
Vương Quân Hoàng \\ Tạp chí Ứng dụng toán học \\ Tập II, số 1, 2004, tr. 15-30 \\ (Ấn phẩm của Hội Toán học Việt Nam) \\ Ngày 15 tháng 6 năm 2004 \\ Hà Nội, Việt Nam
}




\title{
HIỆU ÚNG GARCH TRÊN DÃY LỢI SUÂT: THI TRƯỜNG CHỨNG KHOÁN VIẸT NAM 2000-2003
}

\author{
VƯƠNG QUÂN HOȦNG \\ Centre Emile Berheim. 21 Ave. F.D. Roosevelt, Brussels B-1050
}

\section{Người giới thiệu: Nguyễn Văn Hữu}

TÓM TẢT. Thị trường chứng khoán Việt Nam (TTCKVN) ra đời ngày 20/7/2000, ban đầu được xem là thử nghiệm, theo nghĩa thị trường phài chấp nhận những điểu chinh, hạn chế theo đặc trưng riêng. Bài viết này là một trong những nghiên cứu thống kê ứng dụng bước đầu tim kiểm bẳng chứng khoa học về hiệu ứng $\mathrm{GARCH}$ trèn dãy thống kê lợi suất của chi số giá thị trường vả 10 cồ phiếu đang niêm yết. Kết quả kiểm định đáng khich lệ. Trước tiên, một số kết quả tương đối sát với những bằng chứng trong các báo cáo khoa học khác cho những thị trường phát triển. Sau đó, ta thấy với công cụ phân tích thống kê cho hệ thống kinh tế động, một thị trường dủ non trẻ cũng mang những đặc tính đáng lưu ý trong quá trình ra chinh sảch quản lý. Qua đó, ta rút ra được những nhận định ban đầu, không tầm thường về thị trường chứng khoán (TTCK) sơ sinh, có tuổi đời còn non trẻ ở nước ta. Nghiên cứu còn góp phần làm rõ tầm quan trọng của ửng dụng thống kê toán học, một vấn đề chưa được chú ý nhiều trong giới nghiên cứu khoa học kinh tế ờ Việt Nam.

Tì khoá: GARCH, Thị trường sơ sinh, Kinh tế chuyển đổi, Chuỗi thời gian tài chính.

\section{Tóm tắt về TTCK Việt Nam}

Việt Nam mới bắt đầu quá trình cải cách kinh tế sâu rộng khoảng 17 năm lại đây. Nhiểu thay đổi quan trọng được tiến hành, chuyển dần nền kinh tế sang mô hinh thị trường, trong số đó phải kể đến cải cách hệ thống ngân hàng, bồ sung các thành phần của thị trường tài chính và vốn, trước đó chưa từng tồn tại trong nền kinh tế bao cấp. Một bước tiến quan trọng, có ý nghĩa đột phá chính sách nữa đối với quá trình tự do hoá tài chính là xây dựng Trung tâm giao dịch (TTGD) chứng khoán TP. Hồ Chí Minh. Sự ra đời của TTCKVN là kết quả của một kế hoạch lâu dài tới 7 năm. Với trình độ phát triển kinh tế nhìn chung ở mức thấp, các

\footnotetext{
'Bài viết này dựa trên kết quả tác giả trình bày tại Hội thảo khoa học của Trung tâm nghiên cứu khoa học kinh tế Centre Emile Berheim (CEB), tháng 10/2002, sau đó được hiệu chinh, bổ sung nhờ nhiều đóng góp và nhận xét từ các nhà nghiên cứu và đồng nghiệp. Đặc biệt, tôi xin chân thành cảm ơn các nhà nghiên cứu André Farber, Ariane Szafarz, Pierre-Yves Preumono, Marie-Paule Laurent, Kim Oosterlinck, Jim Riedel, Nguyền Văn Hữu, Trần Hùng Thao, Hà Huy Toàn.
} 
cấu thành kinh tế thị trường được "gieo hạt" chưa đến 2 thập kỷ, nhìn chung, hiểu biết về hoạt động đầu tư trên thị trường tài chính của công chúng còn chưa cao, ngay cả đối với những người điều hành doanh nghiệp. Nghiên cứu này ứng dụng phương pháp phân tích thống kê trên chuỗi thời gian quan sát trên TTGD.

\subsection{Các công ty niêm yết}

Công ty niêm yết là một trong những nhân tố quan trọng nhất của TTCK, đặc biệt trong bối cảnh Việt Nam nhiều công ty không muốn và cũng không sẵn sàng niêm yết. Hai công ty niêm yết đầu tiên là Công ty Cổ phần cơ điện lạnh (REE) và Công ty Vật liệu cáp viễn thông Sài Gòn (SAM), giao dịch ngày 28/7/2000. Tiếp theo là Công ty Cồ phần giấy Hải Phòng (HAP) và Công ty Dịch vụ vận tải xuất nhập khẩu Sài Gòn (TMS), 4/8/2000. Các công ty khác được quan sát trong nghiên cứu này là Công ty Chế biến lương thực Long An (LAF; 15/12/2000), Khách sạn Sài Gòn (SGH; 16/7/2001), Công ty Đồ hộp Hạ Long (CAN; 22/10/2001), Công ty Nhựa Đà Nẵng (DPC; 28/11/2001), Công ty Đường Biên Hoà (BBC; 19/12/2001), Công ty Nước giải khát và Thực phẩm Sài Gòn (TRI; 28/12/2001). Một số thông số cơ bản về các công ty này được tóm tắt trong Bảng 1 .

Bảng 1: Thống kê doanh nghiệp

\begin{tabular}{|c|c|c|c|c|c|c|}
\hline Cty & $\begin{array}{r}\mathrm{SCP}(1) \\
(2) \\
\end{array}$ & $\begin{array}{r}\text { D.thu } \\
2002 / 01 \\
\end{array}$ & $\begin{array}{l}\text { L.nhuận } \\
\text { 2002/01 }\end{array}$ & $\begin{array}{r}\text { C.nợ } \\
\text { 2002/01 } \\
\end{array}$ & $\begin{array}{c}\text { TTS } \\
2002 / 01 \\
\end{array}$ & $\begin{array}{r}\text { C.tức } \\
2002 / 01 \\
\end{array}$ \\
\hline \multirow[t]{2}{*}{ REE } & 22.483 .891 & 411,8 & 33,9 & 169,3 & 443,0 & 1,2 \\
\hline & 15.000 .000 & 293,5 & 84,1 & 65,8 & 330,5 & 1,5 \\
\hline \multirow[t]{2}{*}{ SAM } & 24.006 .700 & 258,5 & 58,0 & 33,3 & 232,2 & 1,3 \\
\hline & 12.000 .000 & 167,2 & 34,8 & 10,2 & 183,1 & 1,5 \\
\hline \multirow[t]{2}{*}{ HAP } & 1.992 .930 & 93,9 & 8,9 & 13,8 & 70,5 & 3,0 \\
\hline & & 80,5 & , & 9,1 & 29,7 & 3,4 \\
\hline \multirow[t]{2}{*}{ TMS } & 2.191 .000 & 78,0 & 8,3 & 58,8 & 99,3 & 1,5 \\
\hline & .000 & 68,3 & 9,4 & 32,0 & 66,4 & 1,8 \\
\hline \multirow[t]{2}{*}{ LAF } & 1.782 .970 & 259,2 & 8,2 & 46,0 & 80,7 & 1,1 \\
\hline & .840 & 212,3 & 3, & 27,6 & 97,5 & 1,2 \\
\hline \multirow[t]{2}{*}{ SGH } & 1.766 .300 & 12,2 & 2,5 & 2,0 & 24,1 & 0,9 \\
\hline & 1.500 .000 & 10,5 & 1,6 & 1,5 & 22,8 & 0,9 \\
\hline \multirow[t]{2}{*}{ CAN } & 3.370 .800 & 111,4 & 7,6 & 38,0 & 80,5 & 1,6 \\
\hline & 3.500 .000 & 181,0 & 8,5 & 26,1 & 65,3 & 1,8 \\
\hline \multirow[t]{2}{*}{ DPC } & 1.587 .280 & 59,5 & 2,4 & 7,8 & 27,0 & 1,0 \\
\hline & 1.523 .000 & 55,5 & 2,6 & 9,5 & 28,2 & 1,0 \\
\hline \multirow[t]{2}{*}{$\mathrm{BBC}$} & 5.343 .000 & & & & 162,9 & 1,0 \\
\hline & 5.600 .000 & 183,2 & 5,8 & 80,3 & 107,2 & 1,0 \\
\hline \multirow[t]{2}{*}{ TRI } & 3.753 .100 & 57,2 & 3,7 & 39,4 & 93,7 & 1,0 \\
\hline & 3.790 .300 & 166,8 & 10,4 & 32,3 & 86,9 & 1,2 \\
\hline \multicolumn{7}{|c|}{$\begin{array}{l}\text { Chú thích. } \mathrm{SCP}(1) \text { : Số lượng cổ phiếu đang lưu hành tại thời điểm dừng } \\
\text { của số liệu; } \mathrm{SCP}(2) \text { : Số lượng cồ phiếu thời điểm niêm yêt; Điểm dừng số } \\
\text { liệu thống kê: } 2 / 7 / 2003 \text { : Giá/cố tức lấy đơn vị } 1.000 \mathrm{VND;} \mathrm{D.thu,} \\
\text { L.nhuận, TTS tính theo tỷ VNĐ. }\end{array}$} \\
\hline
\end{tabular}


Tại thời điểm 4/7/2003 (tức phiên giao dịch 574), TTCKVN có 21 công ty niêm yết, tổng giá trị vốn hoá theo thị giá khá khiêm tốn, xấp xỉ 2,500 tỷ VNĐ.

\subsection{Vài nét về công chúng đầu tư}

Bốn nhóm đầu tư ở TTGD là: (a) tư nhân Việt Nam; (b) tư nhân nước ngoài; (c) công ty trong nước; và $(\mathrm{d})$ nhà đầu tư tồ chức nước ngoài. Thực tế, nếu theo cách phân loại khác, có thể có nhiều nhóm công chúng đầu tư khác nữa. Tuy nhiên, 4 nhóm trên cũng đại diện đủ cho tình hình đầu tư hiện tại. Theo báo cáo của UBCKNN, hiện có xấp xỉ 12.000 tài khoản kinh doanh chứng khoán hợp lệ. Tuy nhiên, thực tế không tới 2.000 tài khoản có giao dịch. Trên $95 \%$ nhà đầu tư là tư nhân trong nước. Pháp nhân kinh doanh chủ yếu là ngân hàng thương mại, công ty bảo hiểm, và một số công ty lớn có nhu cầu phân tán rủi ro, và thử nghiệm cơ cấu đầu tư mới. Nhà đầu tư có tổ chức nước ngoài chủ yếu là công ty bảo hiểm nhân thọ, quĩ đầu tu đóng: Prudential, Kamm Investment, Vietnam Enterprise Invesment Ltd., Vietnam Frontier Fund.

\subsection{Kỹ thuật giao dịch và giơri hạn}

Từ 1/3/2002 TTCK mở cửa tất cả các ngày làm việc. Trước đó, có 3 phiên giao dịch mỗi tuần, thứ 2,4 và 6 . Giao dịch từ 9:00 đến 10:00 trước ngày 20/5/2003. Từ 20/5/2003 kỹ thuật thay đồi: (a) Giao dịch kéo dài tới 10:30; (b) Giao dịch thoả thuận thực hiện riêng ở 30 phút cuối; (c) Hai lần khớp lệnh trong 1 phiên giao dịch (trước đó duy nhất 1 lần). Dạng giao dịch chủ yêu là khớp lệnh qua hệ thống, thông qua hàng chờ lệnh Mua/Bán. Khớp lệnh nghĩa là sử dụng qui tắc ưu tiên để chọn lệnh Mua/Bán nào được thực hiện trong phiên. Các qui tắc ưu tiên là: (i) giá tốt nhất; (ii) khối lượng hợp lệ lớn nhất; (iii) lệnh đặt trước được phục vụ trước; (iv) ưu tiên cá nhân cao hơn tồ chức. Chi có một mức giá đóng cửa trong một phiên giao dịch, được dùng làm tham chiếu cho phiên giao dịch tiếp theo, vì hiện tại mức giới hạn dao động giá vẫn tồn tại, và mức tham chiếu này cho biết giá cao nhất/thấp nhất hợp lệ của phiên ngay sau là bao nhiêu. Vượt khỏi sàn và trần này, lệnh sẽ rơi vào nhóm không hợp lệ. Cũng từ 20/5/2003, lệnh mới ATO (At-the-Open) được đưa vào sử dụng, theo đó nhà đầu tư không bắt buộc đặt mức giá trung bình (kỳ vọng). Giá đóng cửa tự động có hiệu lực với lệnh ATO được khớp.

TTCKVN cũng có nhiều giới hạn kỹ thuật. Nhìn chung, trong thống kê tài chính, các giới hạn của thị trường có nhiều ảnh hưởng tới đặc tính tài sản tài chính, chủ yếu là:

- Biên độ giao động giá: Là giới hạn kỹ thuật có tác động mạnh nhất trên TTCKVN, và nhìn chung trên thế giới. Sử dụng giới hạn để nắn hành vi thị trường. Trong lý thuyết tín hiệu kinh tế, sữ dụng biên độ có 2 mặt. Nếu phù hợp, có thể giúp hạn chế rủi ro trong các điều kiện phát sinh bất ổn đột ngột. Nêu lạm dụng, sẽ phát sinh các yếu tố đầu cơ. Tình hình thay đồi giới hạn giá được tóm tắt ở Bảng 2.

- Một số giới hạn khác: giới hạn khối lượng đặt lệnh, ví dụ vào tháng $6 / 2001$, nhà đầu tư không được mua quá $2.000 \mathrm{CP}$ của một công ty trong một phiên, gây sụt đột ngột lượng đặt mua, kéo theo sụt tổng cầu và chỉ số VNI trong 3 tháng liển, mất $60 \%$ giá trị so với trước khi giới hạn áp dụng. Trên quan điểm tài chính ứng dụng, các giới hạn này, từ khi được đề xuất đã cấu thành dòng thông tin; bản chất thay đồi hành vi đầu tư. 


\subsection{Quan sát thị trường sau 3 năm}

TTCKVN chứng kiến nhiều thay đổi lên xuống khá bất thường. Đầu tiên là giai đoạn tăng nhanh cho tới phiên giao dịch S.137 (26/7/2001), chi số giá VNI đạt 571.04 điểm, mức đỉnh điểm. Khoảng S.138-180 (5/10/2001), VNI rơi tụ do xuống múc thấp kỷ luc đầu tiên 203.12 điểm, mất $64.43 \%$ giá trị sau 14 tuần. Giai đoạn phục hồi khiêm tốn tiếp theo đó khoảng 6 tuần (S.181-199) giúp chỉ số tăng $48 \%$ so với S.180. Biến động tồng quan 3 năm cho trong Hình 1.

Hinh 1: Chì số VNI PGD.1-574

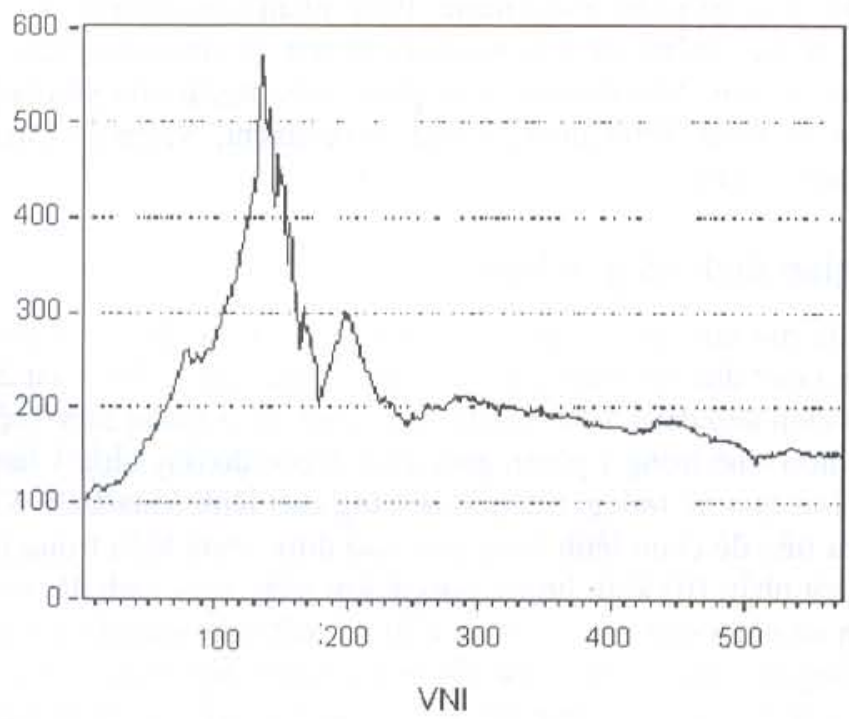

VNI vào PGD.1 là 100. Nguồn@2003 Mezon Finance

Giai đoạn tiếp theo liên tục phá kỷ lục rớt giá. Mức 180.73 điểm (11/3/2002), rồi 139.64 (1/4/2003). Bên cạnh suy giảm giá (kéo theo lợi suất phiên), quan sát cho thấy khối lượng giao dịch cũng không tăng trưởng, gọi là trạng thái lạnh. Các mô hình kinh tế lượng và kiểm định cho biết việc giảm cả giá lẫn khối lượng giao dịch trực tiếp quan hệ với dòng thông tin, thường là tỉn xấu.

\section{Vài nét về kiểm định TTCK}

Hệ thống lý thuyết và kiểm định khoa học trên số liệu TTCK thế giới rất lớn. Phần này tóm tắt hướng chính trong công tác kiểm định thống kê, phục vụ các quyết định quản lý và phát triển kinh tế, liên quan tới tình hình Việt Nam: (i) Hành vi vận động của các biến quan trọng nhất; (ii) Đặc tính thị trường trong nền kinh tế chuyển đổi; và (iii) Hiện tượng phương sai thay đổi phu thuộc trên chuỗi thời gian chứng khoán, đặc biệt lợi suất ngày. 
Bảng 2: Lịch sử và nguyên nhân thay đổi biên độ dao động giá

\begin{tabular}{|c|c|c|c|}
\hline Ngày hiệu lực & Phiên & Biên độ & Nguyên nhân \\
\hline $20 / 7 / 2000$ & S.0 & $(+/-) 5 \%$ & Giữ mức thay đổi giá dao động đủ nhỏ, tránh sốc. \\
\hline $1 / 8 / 2000$ & S.2 & $(+/-) 2 \%$ & $\begin{array}{l}\text { TTGD lo lắng về đám đông đầu tư và sức mua vượt } \\
\text { quá xa khối lượng sắn sàng bán. }\end{array}$ \\
\hline $13 / 6 / 2001$ & S.132 & $(+/-) 7 \%$ & $\begin{array}{l}\text { TTGD muốn khăng định thị trường đủ điều kiền để } \\
\text { vận hành trơn tru, và nhà đẩu tư chịu trách nhiệm về } \\
\text { hành vi đầu tư. Nới rộng biên độ để tăng quyền tự } \\
\text { chủ. }\end{array}$ \\
\hline $10 / 10 / 2001$ & S. 182 & $(+/-) 2 \%$ & $\begin{array}{l}\text { Điều chinh sau gần } 4 \text { tháng sụt giàm sức mua và giá } \\
\text { trên toàn thị trường, ngay sau đỉnh chì số VNI là } 571 \\
\text { điểm, vào tháng } 6 / 2001 \text {. }\end{array}$ \\
\hline $1 / 8 / 2002$ & S.346 & $(+/-) 3 \%$ & $\begin{array}{l}\text { Điều chỉnh để tìm kiếm sôi động sau nhiều tháng } \\
\text { giao dịch khối lượng thấp, và mức cung cồ phiếu } \\
\text { tăng nhanh do thêm công ty niêm yết. }\end{array}$ \\
\hline $2 / 1 / 2003$ & S. 454 & $(+/-) 5 \%$ & $\begin{array}{l}\text { Lý do không rõ. Được coi là một trong nhiều biện } \\
\text { pháp thay đổi kỹ thuật nhằm tăng sức hút thị trường. }\end{array}$ \\
\hline
\end{tabular}

Các lĩnh vực vừa nêu rất rộng. Những nghiên cứu ban đầu phải nhắc tới Cowles [6], khẳng định giá cổ phiếu theo hành trình dao động ngẫu nhiên, vì thế dự báo chúng không hiệu quả. Tiếp theo, Mandelbrot [14] và Fama [8], đưa ra bằng chứng lợi suất cồ phiếu không theo phân phối Gauss như quan niệm ban đầu, mà thường leptokurtic. Theo các nhà khoa học này, lớp phân phối ổn định hợp lý hơn là Pareto, trong đó, phân phối chuẩn là một trường hợp giới hạn. Một tính chất quan trọng trong tài chính ngày nay là tính hiệu quà cùa thị trường, được Fama đưa ra trong [9], phân tích trực tiếp trên dãy lợi suất liên tục. Gần đây, công trình [4] tiếp tục vấn đề lợi suất kỳ vọng và thực tế, trong quan hệ với khối lượng giao dịch, và điểu kiện tồn tại tương quan sai số.

Nhiều nghiên cứu còn cung cấp bằng chứng về TTCK các nước đang phát triển, tính chất các biến kinh tế liên quan trong các môi trường vi mô khác nhau. Trong khu vực, Chang và Ting [5] đề cập đến giao dịch ở Đài Loan dựa trên kiểm định tính hiệu quả. Đặc biệt, Berkman và Lee ([2]) nghiên cứu ảnh hưởng một phần của qui tắc giao dịch, bao gồm cả biên độ giá, lên hành vi thị trường. Xu [20] tổng kết về TTCK Trung Quốc, với nhiều đặc tính được xem là gần gũi với TTCKVN, trên quan điểm cấu trúc vi mô.

Khu vực ASEAN được đề cập đến trong Malliaropulos và Priestley [13]. Tuy nhiên, rất hiếm nghiên cứu về các thị trường tài chính Việt Nam. Gần đây, một trong các nghiên cứu ban đầu [19] phân tích một số đặc tính thống kê của thị trường sơ sinh Việt Nam, trong đó biến nhị phân về tin tốt và xấu được đưa vào nhằm phân tích ảnh hưởng chính sách nói chung. Tuy nhiên, các kết quả lúc đó khá hạn chế, một phần do mẫu thông tin nhỏ. Trong một hội thảo tại trường Đại học Kinh tế Quốc dân Hà Nội (4/2002). GS. A. Farber [10] trực tiếp đề cập đến quá trình điều khiển phản hồi trong hệ động học lợi suất giao dịch ngày cổ phiếu, và cung câp một số bằng chứng ban đầu về sai số phụ thuộc. Các cơ sở phân tích và bằng chứng kiềm định cho hiện tượng phương sai thay đồi phụ thuộc liên quan đến chuỗi 
thời gian tài chính liên tục như lạm phát, giá và lợi suất chứng khoán, tỳ giá hối đoái. v.v.. được trình bày trong [7]. Mô hình mở rộng tiếp theo, cũng là hướng ứng dụng của nghiên cứu này, có thể xem trong [3], [17], [1], [16]. Trên góc độ kiểm định thống kê, một trong những ứng dụng của TTCKVN được trình bày trong [18].

\section{Phương pháp kinh tế lượng và só liệu}

Một số tài liệu về phương pháp và kỹ thuật kiểm định là $[12,11,15]$. Trong các nghiên cứu trên các số liệu tài chính, việc kiểm tra tính ổn định là bước hết sức quan trọng, trước khi tiến hành hồi qui theo mô hình thống kê. Dưới đây chúng ta sử dụng kiểm định Dickey-Fuller $(\mathrm{ADF})$ và Phillips-Perron $(\mathrm{PP})$.

\subsection{Tính ơn định}

Tính ổn định của chuỗi thời gian theo kiểm định $\mathrm{ADF}$ được tìm kiếm trên điều khiển phản hồi

$$
x_{t}=\beta_{0}+\beta_{1} x_{t-1}+\varepsilon_{t} \text {. }
$$

Viết lại dạng sai phân, ta có

$$
\Delta x_{t}=\beta_{0}+\gamma_{1} x_{t-1}+\varepsilon_{t},
$$

trong đó $\gamma_{1}=\beta_{1}-1 ; \varepsilon \sim N(0,1)$.

Giả thiết gốc: $H_{0}: \gamma_{1}=0$, và giả thiết đối: $H_{A}: \gamma_{1}<0$. Dickey và Fuller (1979) cũng chứng minh rằng thống kê $t$ cho $\gamma_{1}$ không theo phân phối Student $t$ truyền thống. Do đó, giá trị tới hạn đã được mô phỏng lại (Dickey-Fuller, 1979; MacKinnon, 1991).

\subsection{Phán hời tự tương quan và sai só tương quan}

Đây là hai hiện tượng phổ biến trong các kiểm định chuỗi thời gian tài chính. Hệ tự tương quan là hệ trong đó giá trị trễ có mặt trong quá trình ngẫu nhiên ở vế phải phương trình hồi qui:

$$
x_{t}=\beta_{0}+\sum_{i=1}^{p} \beta_{i} x_{t-i}+\varepsilon_{t} .
$$

Phương trình (3) thường được dùng mô tả động học của sai số của hệ hồi qui như sau:

$$
u_{t}=\rho_{1} u_{t-1}+\rho_{2} u_{t-2}+\ldots+\rho_{p} u_{t-p}+\varepsilon_{t} .
$$

Phương trình (4) cũng là động học biểu diễn sai số tương quan, nếu một trong các hệ số có ý nghĩa thống kê, dẫn đến ước lượng bình phương nhỏ nhất bị chệch và phi tuyến, và độ lệch chuẩn ước lượng không phù hợp để kiểm định theo các giá trị tới hạn truyền thống. Một trong các phương pháp khử ảnh hưởng này là tăng số biến trễ có mặt ở vế phải của động học. Phương pháp kiểm định cho (4) thường dùng trong tài chính là các phương pháp Breusch- 
Godfrey (BG) và Ljung-Box (LB). BG thiết kế trên thống kê nhân tử Lagrange: $B G=N \cdot R^{2}$. Có thể chứng minh rằng $B G \sim \chi_{k}^{2}$ với $H_{0}: \rho_{1}=\rho_{2}=\cdots=0$. Kiểm định LB xây dựng thống kê $Q$ như sau:

$$
Q_{L B}=T(T+2) \sum_{j=1}^{k} \frac{\tau_{j}^{i}}{T-j},
$$

trong đó $\tau_{j}$ là hệ số tương quan thứ $j ; T$, số quan sát thống kê. Cả hai thống kê đều dùng tốt trong trường hợp biến trễ tồn tại trong động học, khác với Durbin-Watson, chỉ thích hợp cho hệ giải thích bằng biến ngoại sinh, và không tồn tại biến trễ bên vế phải.

\subsection{Phương sai thay đới phụ thuộc-GARCH}

Hiệu ứng GARCH có ý nghĩa giải thích quan trọng đối với hành vi rủi ro của tài sản chính, và là một trong những phát hiện quan trọng của lý thuyết thống kê tài chính trong những năm 80. Xét quá trình tự hồi qui bậc $p$, biến phụ thuộc $x_{t}$ :

$$
x_{t}=c+\phi_{1} x_{t-1}+\phi_{2} x_{t-2}+\cdots+\phi_{p} x_{t-p}+u_{t},
$$

trong đó $u_{t}$ là dãy nhiếu ồn trắng (W.N.) sao cho

$$
\begin{gathered}
E\left(u_{t}\right)=0, \\
E\left(u_{t} u_{\tau}\right)= \begin{cases}\sigma^{2}, & t=\tau, \\
0, & t \neq \tau .\end{cases}
\end{gathered}
$$

Loại quá trình ngẫu nhiên này có mặt khắp nơi trong lý thuyết và kiểm định tài chính. Tuy nhiên, diễn biến của $u_{t}$ cũng có thể dẫn đến $u_{t}^{2} \sim A R(\mathrm{~m})$ :

$$
u_{t}^{2}=\beta_{0}+\beta_{1} u_{t-1}^{2}+\beta_{2} u_{t-2}^{2}+\cdots+\beta_{m} u_{t-m}^{2}+\varepsilon_{t}
$$

trong quá trình động học mới này, $\varepsilon_{t} \sim$ W.N. :

$$
\begin{gathered}
E\left(\varepsilon_{t}\right)=0, \\
E\left(\varepsilon_{t} \varepsilon_{\tau}\right)= \begin{cases}\lambda^{2}, & t=\tau, \\
0, & t \neq \tau,\end{cases}
\end{gathered}
$$

trong đó $E(\cdot)$ là toán từ kỳ vọng. Nếu $u_{t}$ thoả mãn điều kiện (7) thì được gọi là quá trình ARCH bậc $m: u_{t} \sim A R C H(\mathrm{~m})$. Một điều kiện bồ sung để $u_{t}^{2}$ ồn định là

$$
1-\beta_{1} z-\beta_{2} z^{2}-\cdots-\beta_{m} z^{m}=0
$$

có nghiệm với mô-đun lớn hơn 1 . Ký hiệu $\hat{u}_{t+s \mid t}^{2}$ là một dự báo tuyến tính ngoài mẫu cho $s$ giai đoạn tiếp theo: 


$$
\hat{u}_{t+s \mid t}^{2}=E\left(u_{t+s}^{2} \mid u_{t}^{2}, u_{t-1}^{2}, u_{t-2}^{2}, \ldots\right) .
$$

Nếu $\operatorname{Var}\left(\varepsilon_{\mathrm{t}}\right)<\infty$, ta có

$$
\hat{u}_{t+s \mid t}^{2} \stackrel{s \rightarrow \infty}{\longrightarrow} \sigma^{2},
$$

trong đó $\sigma^{2}$ là phương sai không phụ thuộc của $u_{t}$.

Khái niệm $\mathrm{ARCH}$ tổng quát $(\mathrm{GARCH})$ do Bollerslev [3] đưa ra năm 1987. GARCH khắc phục nhược điểm trong mô hình hoá tài chính do qui cách nhiều tham số của $\operatorname{ARCH}(p)$ (bậc $p$ khá cao để triệt tiêu các ảnh hưởng làm méo ước lượng). Qui cách phổ biến nhất trong các kiểm định chuỗi tài chinh là kiểm định mô hình $\operatorname{GARCH}(1,1)$. Giả sử $x_{t}$ (vd., lợi suất ngày chứng khoán) được kiểm định theo (1). GARCH mô tả phân phối phụ thuộc của $\varepsilon_{t}$ như sau:

$$
\operatorname{Var}_{t-1}\left(x_{t}\right)=E_{t-1}\left(\varepsilon_{t}^{2}\right)=\sigma_{t}^{2} .
$$

Phương sai phụ thuộc cho biết sự phụ thuộc vào các quan sát thống kê trong (9). Quá trinh $\operatorname{GARCH}(p, q)$ mô tả như sau:

$$
\sigma_{t}^{2}=\kappa+\sum_{i=1}^{p} \alpha_{i} \sigma_{t-i}^{2}+\sum_{j=1}^{q} \gamma_{j} \varepsilon_{t-j}^{2}
$$

trong đó $\sigma_{t}^{2}$ cho biết kỳ vọng của phương sai giai đoạn kế tiếp; $\sigma_{t-i}^{2}, i=1, \ldots, p$, là dãy $p$ phương sai trong quá khứ dự báo từ mô hình $\varepsilon_{t-j}^{2}, j=1, \ldots, q$, là $q$ phương sai quan sát thực. Như vậy, khi cho phần tử GARCH $p=0$, mô hình rút gọn về $\mathrm{ARCH}(q)$, một trường hợp của GARCH tồng quát.

Biến ngoại sinh và änh hường chinh sách:

Trên thực tế, các biến ngoại sinh có thể cho biết các tác động chính sách tới hệ thống động học, giải thích một số thay đổi đột biến trong diễn biến chuỗi thời gian. Trong trường hợp Việt Nam, các biến này bao gồm qui tắc giao dịch, thay đồi kỹ thuật, và các hạn chế mới của cơ quan điều hành, như quá trình

$$
\sigma_{t}^{2}=\kappa+\alpha_{1} \sigma_{t-1}^{2}+\alpha_{2} \sigma_{t-2}^{2}+\cdots+\gamma_{1} \varepsilon_{t-1}^{2}+\gamma_{2} \varepsilon_{t-2}^{2}+\cdots+\phi_{1} z_{1 t}+\phi_{2} z_{2 t},
$$

trong đó $z_{1 t}$ có thể đại diện cho mức giới hạn biên độ giá; $z_{2 t}$ dòng tin tốt về lợi nhuận doanh nghiệp, mức chi trả cồ tức, v.v..

\section{Kết quả kiểm định và ước lượng}

Nghiên cứu ước lượng và kiểm định trên TTCKVN giai đoạn 2000-2003 (hơn 35 tháng). Điểm dừng số liệu là $5 / 7 / 2003$, phiên giao dịch S.574. Tổng mức chênh lệch lượng đặt mua 
và bán theo phiên cho ở Hình 2 , cũng là một thành phần quan trọng trong kiến thức của chúng ta về hành vi thị trường.

Hình 2: Dư mua tổng thị trường PGD.1-574

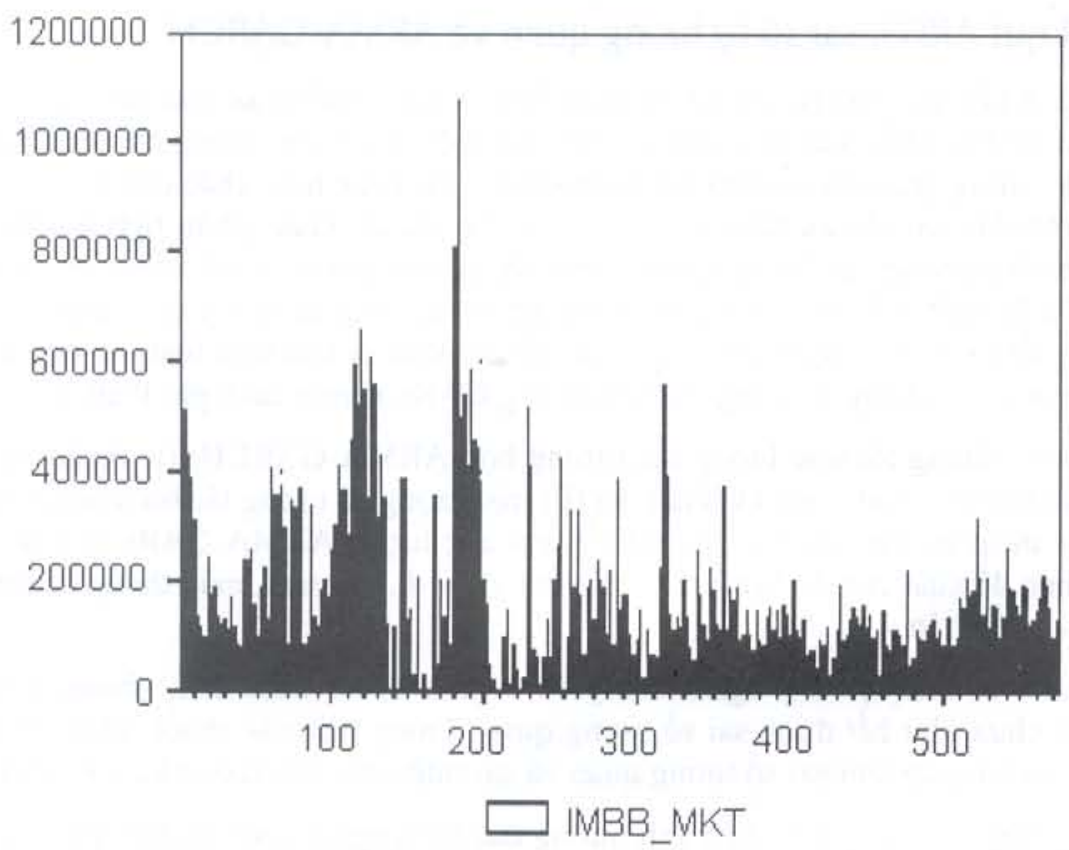

\subsection{Kiểm định ADF, PP và qui cách gián đơn của phản hời tự hời qui}

Sử dụng phương pháp $\mathrm{ADF}$ và $\mathrm{PP}$ đã đề cập ở trên ${ }^{2}$, chúng tôi thu được kết quả rõ rệt cho thấy tất cả các dãy lợi suất đều ồn định và có tính dừng khá tốt, căn cứ trên ước lượng $\mathrm{ADF}$ và PP có giá trị tuyệt đối chạy từ 10.56 tới 20.58 , tuỳ thuộc vào đối tượng và độ lớn của mẫu. Trong khi đó, so sánh với giá trị tới hạn, phổ biến dao động xung quanh mức 3.44. Như vậy, giả thiết gốc không dừng bị bác bỏ ở mức $1 \%$. Việc sử dụng các chuỗi này trong các hồi qui tuyến tính là phù hợp cho suy luận thống kê. Ngoài ra, các thông số khác của kiểm định cũng khá tốt, chẳng hạn $\bar{R}^{2}$ ở mức 35-40\%, và $L(\theta)$ chạy trong khoảng 1100 tới 1300 .

Tiếp theo, nghiên cứu xem xét các dấu hiệu của tự tương quan có thể nhận biết bằng thống kê Ljung-Box $Q$ cho tất cả 10 cổ phiếu và chi số VNI toàn thị trường, và đồng thời còn xét tới độ lớn của 2 hệ số căn bản khác là $A C F$ và $P A C F$. Tính chất dễ quan sát thấy là sự hiện diện của tự tượng quan sai số rất rõ rệt, cho tới tận giá trị trễ 35 , với tất cả các xác

${ }^{2}$ Để tránh đưa các hệ thống bảng biểu thuần tuý thống kê, trong bài viết tập trung vào phân tích ý nghĩa của kết quả kiểm định, cũng như tính phù hợp. Độc giả quan tâm đến chi tiết quy cách kỹ thuật, đề nghị liên hệ trực tiếp với tác già. 
suất khử $\mathrm{p}$-Val bằng 0 . Trong khi đó, hệ số $\mathrm{ACF} / \mathrm{PACF}$ của tất cả các biến ở trễ $(t-1)$ đều khá lớn và mang dấu dương, dao động lớn từ 0.20 tới 0.47 . Như vậy, một đặc trưng nữa của chuỗi thời gian tài chính một lần nữa được xác nhận trên TTCKVN.

\subsection{Hồi qui $A R(1)$, sai só tự tượng quan và ARMA-GARCH}

Khi phân tích hồi qui $\mathrm{AR}(1)$, chúng tôi nhận thấy ý nghĩa thống kê của hầu hết tất cả các hệ số của biến trễ bậc nhất, xác định dương. Bên cạnh đó, $4 / 11$ ước lượng cho thấy vẫn còn khả năng tồn tại tương quan sai số theo mô hình $\mathrm{AR}(1)$. Một dấu hiệu khác cho biết sự tồn tại của GARCH trên dãy sai số sau hồi qui khá mạnh. Từ góc độ khác, phân tích hồi qui tự tương quan trên bình phương của lợi suất, tức là mô phỏng gần gũi của mức rủi ro cho thấy $9 / 11$ hệ số biến trễ bậc nhất $\mathrm{AR}(1)$ có ý nghĩa thống kê, trong đó 8 mức ở $1 \%, 2$ mức ở $10 \%$. Tiếp theo, thống kê kiểm tra Engle cho dấu hiệu $A R C H$ đưa ra hầu như toàn bộ đều có ý nghĩa, với $\mathrm{p}-\mathrm{Val}$ nói chung bằng 0 , công nhận hiệu ứng GARCH một cách phi tham số.

Tiếp theo, chúng tôi ước lượng hai trường hợp ARMA-GARCH: (i) thuần tuý GARCH chưa có ảnh hưởng chính sách kỹ thuật: và (ii) ước lượng có tương tác biến ngoại sinh do các thay đổi kỹ thuật tạo ra. Trường hợp (i) kết quả ước lượng ARMA-GARCH trình bày trong Bảng 3. Theo đề xuất của $\mathrm{A}$. Szafarz. A. Farber và $\mathrm{M}-\mathrm{P}$. Laurent, mầu thống kê được rút bớt 100 quan sát đầu tiên.

Tất cả hệ số $\mathrm{AR}(1)$ có ý nghĩa thống kê ở mức cao 1 hoặc $5 \%$, nhưng trong một số trường hợp chưa khử hết được sai số tương quan. Trong một vài chuỗi, phần tử trung bình trượt MA tồn tại giúp khử sai số tương quan và cải thiện chì tiêu thống kê $\mathrm{AIC}(5 / 11)$.

Trường hợp (ii) giúp phân tích ảnh hưởng của biến ngoại sinh lên kết quả ước lượng và giải thích một số hiện tượng của TTCKVN. Các biến bổ sung trực tiếp vào phương trình kỳ vọng và phương sai bao gồm các nhóm thông tin ngoại sinh: $(*)$ biên độ dao động giá: $(* *)$ chênh lệch đặt mua/bán: $(* * *)$ thay đồi kỹ thuật giao dịch. Hệ động học mới, có tính giải thích cao hơn, được cho như sau:

$$
\begin{aligned}
& \Upsilon_{t}=C+\sum_{i=1}^{m} \phi_{i} \Upsilon_{t-i}+\varepsilon+\sum_{j=1}^{n} \theta_{j} \varepsilon_{t-j}+\sum_{k=1}^{0} \zeta_{k} y_{k}, \\
& \sigma_{t}^{2}=\kappa+\sum_{\Upsilon=1}^{p} \alpha_{v} \sigma_{t-v}^{2}+\sum_{\mathrm{w}=1}^{q} \gamma_{\mathrm{w}} \varepsilon_{t-\mathrm{w}}^{2}+\sum_{l=1}^{s} \zeta_{l} y_{l} .
\end{aligned}
$$

Do các phân tích trên thông tin ước lượng nhóm động học kỳ vọng lợi suất khá đơn giản, dưới đây chỉ đưa tóm tắt nhóm ước lượng trên động học phương sai của 5 chuỗi và kiểm định thực hiện trên 475 quan sát (Bàng 4).

Một số kiểm định bồ sung về tính hợp lý của mô hình chứa biến ngoại sinh được thực hiện trên thống kê AIC. Engle LM. JB. $Q$ và $Q^{2}$, tại các trễ $6,12,36$. Các kết quả đều khá tốt, ngoại trừ $Q_{6}^{2}$ của dãy $\mathrm{SAM}$ vẫn còn ý nghĩa thống kê ở mức $5 \%$. 
Bảng 3: Kiểm định mô hình ARMA-GARCH (i)

\begin{tabular}{|c|c|c|c|c|c|c|c|c|c|c|}
\hline Tham số & HAP & & REE & & SAM & & TMS & & VNI & \\
\hline C & -.002888 & & 0.000924 & & 0.000895 & & 0.005828 & & -0.000423 & \\
\hline - s.e. & 0.009979 & & 0.000796 & & 0.000731 & & 0.006205 & & 0.002941 & \\
\hline - zStat & -.289435 & & 1.161253 & & 1.224485 & & 0.939314 & & -0.143917 & \\
\hline AR1 & 1.152647 & $\cdot$ & 0.358806 & $*$ & 1.057342 & * & 1.214548 & * & 1.24649 & • \\
\hline -s.e. & 0.054038 & & 0.044925 & & 0.151074 & & 0.069508 & & 0.172908 & \\
\hline - zStat & 21.33019 & & 7.986831 & & 6.998841 & & 17.47342 & & 7.208993 & \\
\hline AR2 & -.154129 & - & & ¿ & -0.155122 & 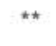 & -.221286 & * & -0.250667 & \\
\hline - s.e. & 0.053713 & & & & 0.07989 & & 0.066283 & & 0.174656 & \\
\hline - zStat & -2.8695 & & & & -1.941682 & & -.338505 & & -1.435204 & \\
\hline MA1 & -0.980379 & $\cdot$ & & & -0.817284 & & -.909783 & $*$ & -0.761138 & * \\
\hline -s.e. & 0.006603 & & & & 0.135379 & & 0.02884 & & 0.131297 & \\
\hline - zStat & -148.4657 & & & & -6.037019 & & -1.54624 & & -5.79705 & \\
\hline kappa & 0.0000206 & $* * *$ & 0.0000193 & $* *$ & 0.00001870 & $* *$ & 0.0000221 & $* *$ & .00000289 & \\
\hline -s.e. & 0.0000113 & & 0.0000085 & & 0.00000868 & & 0.0000101 & & .00000236 & \\
\hline - zStat & 1.820879 & & 2.278195 & & 2.156004 & & 2.177896 & & 1.225362 & \\
\hline ARCH1 & 0.216350 & • & 0.339617 & * & 0.419984 & • & 0.303381 & * & 0.302972 & * \\
\hline -s.e. & 0.068175 & & 0.058415 & & 0.087187 & & 0.067850 & & 0.083052 & \\
\hline - zStat & 3.173459 & & 5.813817 & & 4.817028 & & 4.471357 & & 3.647963 & \\
\hline GARCH1 & 0.695349 & * & 0.611902 & $*$ & 0.52237 & - & 0.632065 & ${ }^{*}$ & 0.745075 & - \\
\hline -s.e. & 0.097472 & & 0.072053 & & 0.081695 & & 0.075334 & & 0.0473 & \\
\hline - zStat & 7.133841 & & 8.492334 & & 6.394141 & & 8.390176 & & 15.75195 & \\
\hline LogL & 1642.046 & & 1604.738 & & 1685.101 & & 1646.367 & & 1821.529 & \\
\hline AIC & -.757204 & & -.593488 & & -5.877763 & & -5.77242 & & -6.352114 & \\
\hline Engle LM & 0.757219 & & 0.048839 & & 0.042509 & & 0.004315 & & 0.010758 & \\
\hline$J B$ & 770.50 & & 43.47 & $*$ & 225.50 & • & 721.00 & & 2921.00 & * \\
\hline Q_6 & 2.87 & & 48.426 & $\cdot$ & 12.92 & $\star$ & 6.56 & $* * *$ & 3.71 & \\
\hline Q_12 & 12.16 & & 81.713 & $*$ & 22.52 & $*$ & 9.65 & & 9.98 & \\
\hline Q_36 & 37.93 & & 126.94 & $*$ & 45.94 & $* * *$ & 33.63 & & 33.81 & \\
\hline
\end{tabular}

Ghi chú: Tối ưu hóa tham số trên môi trường EViews (c) 4.1. Quy cách ARMA lựa chọn theo Akaike Information Criterion (AIC) và ước lượng hợp lý cực đại (LogL). ARCHi tương ứng với hệ số $y_{i}$ và $\mathrm{GARCHj}$ tương ứng với hệ số $\alpha_{1}$ trong phương trinh (2). Các ký hiệu *, **, *** có ý nghĩa thống kê ở mức 1,5 và $10 \%$. 
Bảng 4: Kiểm định ARMA-GARCH (ii) - động học phương sai

\begin{tabular}{|c|c|c|c|c|c|c|c|c|c|c|}
\hline Tham số & HAP & & REE & & SAM & & TMS & & VNI & \\
\hline kappa & $-3.27 \times 10^{-6}$ & & $-1.05 \times 10^{-5}$ & & $6.64 \times 10^{-6}$ & & $-8.10 \times 10^{-6}$ & $*$ & $1.72 \times 10^{-6}$ & \\
\hline s.e. & $8.41 \times 10^{-6}$ & & $1.77 \times 10^{-5}$ & & $8.69 \times 10^{-6}$ & & $2.43 \times 10^{-6}$ & & $9.04 \times 10^{-6}$ & \\
\hline zStat & -0.388626 & & $-5.91 E-01$ & & 0.764771 & & -3.333118 & & 0.189850 & \\
\hline $\mathrm{ARCH}(1)$ & 0.145277 & * & 0.244372 & $*$ & 0.238878 & $*$ & 0.423145 & * & 0.264692 & $* *$ \\
\hline s.e. & 0.055166 & & 0.05946 & & 0.087571 & & 0.089641 & & 0.109623 & \\
\hline zStat & 2.633464 & & 4.109867 & & 2.727833 & & 4.720454 & & 2.414580 & \\
\hline $\mathrm{GARCH}(1)$ & 0.596518 & * & 0.574743 & $*$ & 0.669524 & * & 0.590626 & • & 0.543867 & * \\
\hline s.e. & 0.116237 & & 0.129125 & & 0.08363 & & 0.069109 & & 0.164858 & \\
\hline zStat & 5.13189 & & 4.451044 & & 8.005798 & & 8.54635 & & 3.299006 & \\
\hline BBCDB & $-2.70 \times 10^{-5}$ & $* *$ & $-6.58 \times 10^{-5}$ & $* *$ & $-1.30 \times 10^{-5}$ & $*$ & $5.66 \times 10^{-5}$ & & $-5.51 \times 10^{-5}$ & * \\
\hline s.e. & $1.37 \times 10^{-5}$ & & $3.74 \times 10^{-5}$ & & $5.81 \times 10^{-6}$ & & $3.24 \times 10^{-5}$ & & $1.38 \times 10^{-5}$ & \\
\hline zStat & -1.967906 & & -1.757973 & & -2.244237 & & 0.174984 & & -3.986807 & \\
\hline CANDB & $3.89 \times 10^{-5}$ & & $-2.26 \times 10^{-5}$ & & & & $-2.16 \times 10^{-5}$ & $\cdot$ & $2.41 \times 10^{-5}$ & \\
\hline s.e. & $5.79 \times 10^{-5}$ & & $1.68 \times 10^{-5}$ & & & & $6.13 \times 10^{-6}$ & & $4.06 \times 10^{-5}$ & \\
\hline zStat & 0.672484 & & -1.34545 & & & & -3.524398 & & 0.594811 & \\
\hline MKTDB & $-1.43 \times 10^{-5}$ & ** & $-1.33 \times 10^{-5}$ & & & & $2.43 \times 10^{-5}$ & & $-8.36 \times 10^{-6}$ & \\
\hline s.e. & $6.68 \times 10^{-6}$ & & $1.89 \times 10^{-5}$ & & & & $3.86 \times 10^{-5}$ & & $9.03 \times 10^{-6}$ & \\
\hline zStat & -2.136521 & & -0.702134 & & & & 0.628414 & & -0.926465 & \\
\hline BANDN & 0.000656 & & 0.001479 & & $9.27 \times 10^{-5}$ & & 0.00062 & * & 0.000434 & \\
\hline s.e. & 0.000429 & & 0.001108 & & 0.000198 & & $5.04 \times 10^{-5}$ & & 0.000512 & \\
\hline zStat & 1.530014 & & 1.33445 & & 0.467879 & & 12.29297 & & 0.848341 & \\
\hline REESpl & $5.64 \times 10^{-6}$ & & $3.71 \times 10^{-5}$ & & & & & & & \\
\hline s.e. & $3.47 \times 10^{-5}$ & & $7.42 \times 10^{-5}$ & & & & & & & \\
\hline zStat & 0.162578 & & 0.500323 & & & & & & & \\
\hline SAMSpl & $-6.94 \times 10^{-5}$ & * & & & & & & & & \\
\hline s.e. & $2.38 \times 10^{-5}$ & & & & & & & & & \\
\hline zStat & -2.912338 & & & & & & & & & \\
\hline $\log L$ & 1634.182 & & 1394.423 & & 1634.947 & 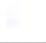 & 1488.437 & & 1587.471 & \\
\hline
\end{tabular}

Nói tóm lại, mô hình chứa biến ngoại sinh khử tự tương quan tốt hơn đáng kể, và loại trừ hoàn toàn hiệu ứng GARCH ở các hệ số trễ thời gian xa hơn. Việc đưa biến ngoại sinh vào đã làm thay đổi đáng kể tính giải thích và động học thu được từ ước lượng. Hầu hết các hệ số $\mathrm{AR}(1)$ không còn ý nghĩa thống kê. Các biến ngoại sinh đã thay thế chúng giải thích các biến động. Hậu tố $s p l$ đại diện cho thông tin tách cổ phiếu. $D B$ đại diện thông tin xấu và $D G$ thông tin tốt, về doanh thu, lợi nhuận, thay đổi nhân sự, triển vọng thị trường. BAND là hệ số giá trần theo ngày. BANDN là biên độ thay đồi giá mức trên. RRMKTV là tốc độ tăng trường của tổng mức giao dịch thực hiện, và RRVNI là lợi suất từ chỉ số giá thi trường. Mô hình chi sử dụng những thông tin được xem là chính thống và quan trọng nhất, ví dụ như Bibica về báo cáo chậm, che dấu lỗ; CAN là vụ gian dối hoàn thuế VAT; REE là thông tin xử lý lại hệ thống hạch toán,... 
Kết quả ước lượng cho thấy các biến ngoại sinh có sức giải thích sinh động hơn và các ảnh hưởng của chúng đa dạng hơn. Chẳng hạn, tin tiêu cực về $\mathrm{BBC}$ và $\mathrm{CAN}$ ành hưởng tới động học phương sai. Thông tin của hai cô phiếu này ảnh hưởng mạnh tới mức rủi ro của các cổ phiếu khác, mặc dù chúng không phải là những cổ phiếu lâu nhất, và giao dịch nhiều nhất. Nói cách khác, sức nặng của thông tin phụ thuộc và thời điểm và tính chất của nó. Bên cạnh đó, tình hình chung của thị trường được phản ánh qua hai dòng thông tin MKTDG và MKTDB, nhìn chung có ý nghĩa thống kê. Mức ảnh hưởng của biên độ giá cũng không thống nhất. Hai sự kiện được công chúng xem là quan trọng, tách cổ phiếu của REE và SAM lại cho thấy mức ý nghĩa thống kê không đáng kể đối với động học rủi ro, nhưng đáng kể đối với kỳ vọng lợi suất của một số cổ phiếu khác $(8 / 11)$. Hình dưới đây mô tả diễn biến của phương sai phụ thuộc từ ước lượng.

Phương sai phụ thuộc VN-Index và SAM
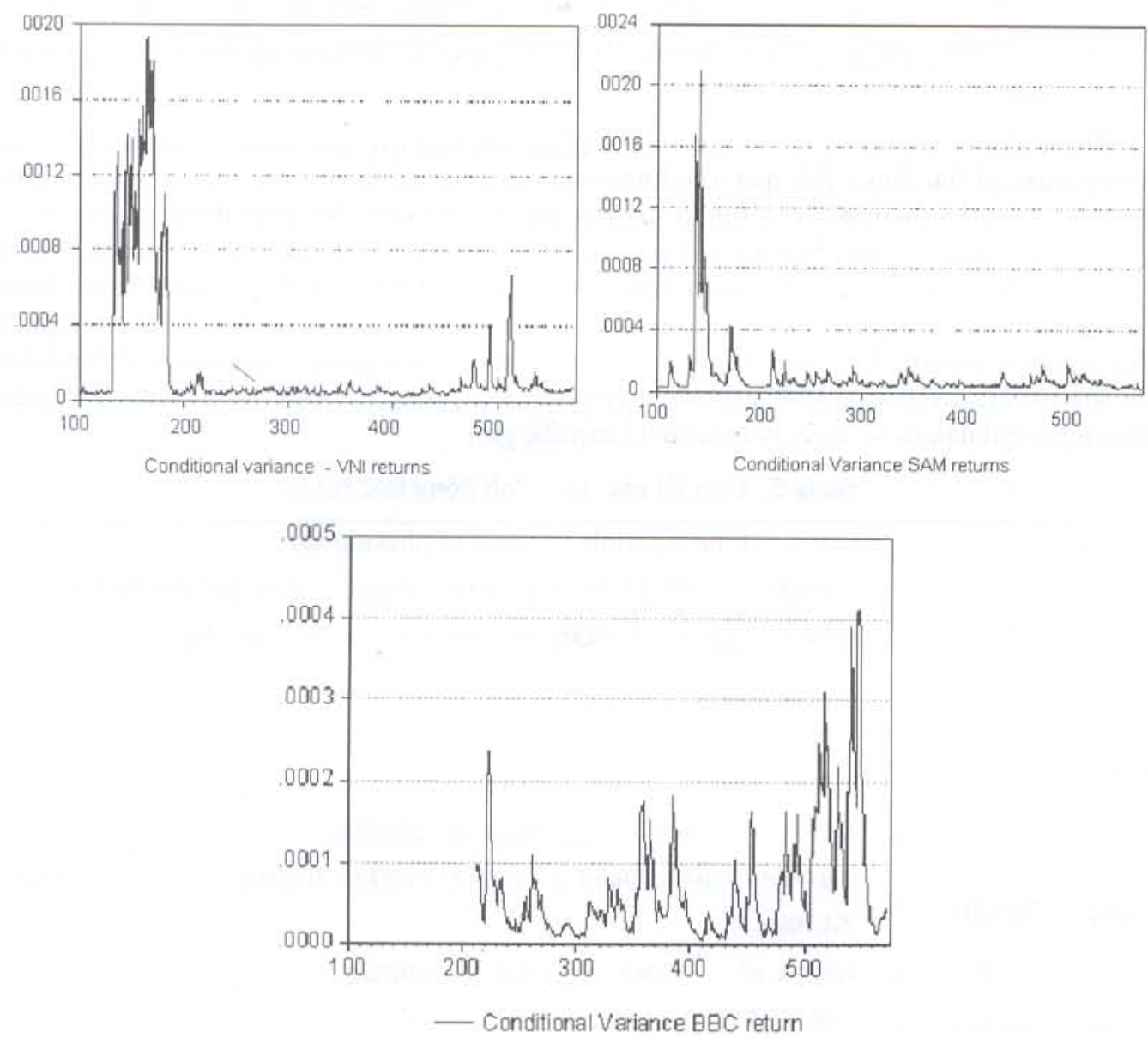

Hình 3. Quá trình phương sai của Bibica

Hai đồ thị trên có dạng tương tự, với hai thời điểm tăng đột ngột mức rủi ro. Tuy nhiên trường hợp của $\mathrm{BBC}$ rất khác biệt. Quá trình động học của phương sai phụ thuộc tỏ ra nhạy 
cảm hơn nhiều và mất ổn định. Xu hướng quan sát được là rủi ro tảng giảm theo các chu kỳ ngắn (xem Mục 4.2).

\section{Một vài nhận xét}

Tới đây, chúng ta có thể đưa ra một vài nhận xét dựa trên ý nghĩa thống kê của rất nhiều ước lượng đã phân tích:

1. Các biến chính sách có ảnh hưởng mạnh, TTCKVN rất nhạy cảm với những chuyển động của cơ quan điều phối là TTGD và UBCKNN. Tác nhân mạnh nhất là biên độ dao động giá.

2. TTCKVN mang đặc trưng chung là chịu ảnh hưởng mạnh của dòng thông tin thị trường; và doanh nghiệp. Tin xấu ảnh hưởng nhanh, mạnh và trực tiếp hơn tin tốt.

3. Một trong những tác động nhanh là tác động qua lại giữa các cổ phiếu, theo kiểu dầu loang.

Dưới đây là biểu diễn quen thuộc dạng động học của các quá trình rủi ro dựa trên ước lượng tham số thu được. Kết quả ước lượng này rất có ý nghĩa với ứng dụng trên thị trường tài chính. Cụ thể, biến đổi của rủi ro thường dẫn tới: (i) thay đổi hoạt động đầu tư, do đó quan hệ cân bằng toàn thị trường; (ii) giải thích chuyển dịch vốn giữa các thị trường; và (iii) ảnh hưởng và hiệu quả của thu nhận dòng thông tin tài chính. Đặc biệt, quan hệ ước lượng cho phép dự báo ngoài mẫu trong giai đoạn ngắn, cung cấp một thông số rất quan trọng cho ứng dụng tối ưu hoá danh mục đầu tư, phân tán rủi ro, theo phương pháp trung bình-phrơong sai Markowitz và Sharpe-Lintner-Mossin, một phương pháp căn bản được sử dụng tại hàng trăm ngàn quĩ đầu tư và công ty tài chính khắp thế giới.

Bảng 5. Tóm tắt các quá trình động học rủi ro

\begin{tabular}{|c|c|c|c|}
\hline Chuỗi & & & Phương trình kỳ vọng và phương sai \\
\hline \multirow[t]{2}{*}{ VNI } & $\Upsilon V N I . t$ & $=$ & $\begin{array}{l}0.11823+0.1287 \cdot \curlyvee \text { VNI.t }-1-0.116 \cdot \text { Band }_{t-1}+8.65 \cdot 10^{-4} R R M k t . t V- \\
4.28 \cdot 10^{-8} \text { Mkt } \operatorname{Im} b+8.485 \cdot 10^{-3} \text { MktDG }-0.01664 \cdot \text { REESpl }\end{array}$ \\
\hline & $\sigma_{i}^{2}$ & $=$ & $0.5439 \cdot \sigma_{l-1}^{2}+0.2647 \cdot \varepsilon_{l-1}^{2}-5.51 \cdot 10^{-5} \cdot B B C D B$ \\
\hline \multirow[t]{2}{*}{ REE } & $\Upsilon$ rEE.t & $=$ & $\begin{array}{l}0.2077+0.0935 \cdot \Upsilon R E E . t-1-0.2052 \cdot \text { Band }_{t-1}-9.98 \cdot 10^{-8} \text { REEIMB }+ \\
0.0093 \cdot \text { MktDG }+0.00786 \cdot \text { REESpl }\end{array}$ \\
\hline & $\sigma_{i}^{2}$ & $=$ & $0.5747 \cdot \sigma_{t-1}^{2}+0.2444 \cdot \varepsilon_{t-1}^{2}-6.58 \cdot 10^{-5} B B C D B$ \\
\hline \multirow[t]{2}{*}{ SAM } & YSAM.t & $=$ & $\begin{array}{l}-0.0658+0.0645 \cdot \text { Band }_{t-1}+1.0742 \cdot \Upsilon V N I . t-0.00609 \cdot M k t D G-0.002123 \\
\text { SAMSpl }\end{array}$ \\
\hline & $\sigma_{t}^{2}$ & $=$ & $0.6695 \cdot \sigma_{t-1}^{2}+0.2389 \cdot \varepsilon_{t-1}^{2}-1.3 \cdot 10^{-5} B B C D B$ \\
\hline \multirow[t]{2}{*}{ HAP } & $\Upsilon H A P . t$ & $=$ & $1.0614 \cdot \Upsilon V N I . t$ \\
\hline & $\sigma_{i}^{2}$ & $=$ & $\begin{array}{l}0.5965 \cdot \sigma_{t-1}^{2}+0.1453 \cdot \varepsilon_{t-1}^{2}-2.7 \cdot 10^{-5} \cdot B B C D B-1.43 \cdot 10^{-5} M k t D B- \\
6.94 \cdot 10^{-5} S A M S p l\end{array}$ \\
\hline
\end{tabular}




\section{TÀI LIỆ DẪN}

[1] Baillie. R., Bollerslev T., Intra-day and inter-market volatility in foreign exchange rates, Review of Economic Studies 58 (1990), 565-585.

[2] BERKMAN H., JOHN BYONG TEK LEE, The effectiveness of price limits in an emerging market: Evidence from korean stock exchange, Pacific-Basin Finance Journal 10 (2002), 517-530.

[3] BOLlersLeV T., A conditionally heteroskedastic time series model for speculative prices and rates of return, The Review of Economics and Statistics 69 (3), Aug. 1987.

[4] Cambell J. Y., Grossman S.J., WANG J., Trading volume and serial correlation in stock returns, The Quarterly Journal of Economics, Nov. 1993, 905-935.

[5] CHANG K.-P., TING K.-S., A variance ratio test of the random walk hypothesis for Taiwan's stock market, Applied Financial Econometrics 10 (2000), 525-532.

[6] CoWles A., Can stock market forecasters forecast, Econometrica 1 (1993), 309324.

[7] ENGLE R.F., Autoregessive conditional heteroscedasticity with estimates of the variance of the united kingdom inflation, Econometrica 50 (4), July 1982.

[8] FAMA E.F., The behavior of stock-market prices, Journal of Buiness 38 (1), Jan, 1965.

[9] FAMA E.F., Efficient capital markets: A review of theory and empirical works, Jounal of Finance 25 (1970), 383- 417.

[10] FARBER A., Vietnam's emerging stock market: Reviews and comments, National Economics Univ. Conference, Apr. 2002.

[11] Gourieroux C., SCAILLET O., SzAFARz A., Économétrie de la finance, école nationale de la Statistique et de l'Administration économique et Centre d'études des Programmes économique, Economica.

[12] hamilton J.D., Time Series Analysis, HapperCollins, 2000.

[13] Malliaropulos D., Priestley R., Mean reversion in southeast asian stock markets, Journal of Empirical Finance 6 (1999), 355-384.

[14] MANDELBROT B., The variation of certain speculative prices, Journal of Business 36 (1963), 394-419.

[15] MILLS T.C., The econometric modelling of financial time series, Cambrigde University Press, Cambridge, UK, 1993.

[16] NELSON D,B., Conditional heteroskedasticity in asset return: a new approach, Econometrica 59 (1991), 347-370.

[17] TSAY R., Conditional heteroscedastic time series models, Journal of American Statistical Association, 1987, 590-604. 
[18] Q-HOANG VUONG, Vietnam's equity market: An investigation of first 26-month opeation, CEB/SBS research seminar paper, Oct. 2002.

[19] Q-HOANG VUONG, S-CHUONG TRAN, Information, returns and risks: an empirical investigation of Vietnam's stock market, Economics and Development Review (National Economics Univ., Hanoi) 7 (5), 2001, 43-48.

[20] Xu C.K., The microstructure of the chinese stock markets, China Economics Review 11 (2000), 79-97.

\title{
THE EMPIRICAL EVIDENCE OF GARCH EFFECTS ON RETURN SERIES: VIETNAM STOCK MARKET 2000-2003
}

\begin{abstract}
The Vietnamese Stock Market was officially born on July 20, 2000, and considered and experiment, in the sense that it would likely accept adjustment and constraints to reflect the contemporaneous national economic settings. This paper is one of the first applied econometric studies investigating an evidence of GARCH effects on return series of 10 individual assets and the VNI, an index devised as the market general market price indicator. The results are encouraging: Firstly, we found evidence that the time series exhibit many similar properties as those for other regional markets, such as autoregressive and serial correlation; Secondly, using rather sophisticated empirical models for a newborn market, we succeed in achieving some nontrivial remarks with respect to the use of policy matters. This paper demonstrates the importance of the application of statistical methods, a topic still not received much attention from the economics researchers in Vietnam.
\end{abstract}

JEL Code: C12: C22.

Keywords: GARCH: Newborn stock market; Transitional economy; Financial time series.

Địa chi tác già:

Vương Quân Hoàng

Centre Emile Berheim, 21 Ave. F.D. Roosevelt, Brussels B-1050

E-mail: qvuong@ulb.ab.be; hoangvq@empirics.net 